\title{
Antiphase boundaries on low-energy-ion bombarded $\mathrm{Ge}(001)$
}

\author{
H.J.W. Zandvliet, E. de Groot \\ Faculty of Applied Physics and Centre of Materials Research, University of Twente, P.O. Box 217, 7500 AE Enschede, The Netherlands
}

Received 26 May 1996; accepted for publication 29 July 1996

\begin{abstract}
Surface vacancy and adatom clusters have been created on $\mathrm{Ge}(001)$ by bombarding the surface with $800 \mathrm{eV}$ argon ions at various substrate temperatures ranging from room temperature to $600 \mathrm{~K}$. The vacancies preferentially annihilate at the ends rather than at the sides of the dimer rows, resulting in monolayer deep vacancy islands which are elongated in a direction of the dimer rows of the upper terrace. As vacancy islands nucleate and expand, the dimer rows in neighbouring vacancy islands need not, in general, align with each other. An antiphase boundary will develop if two growing vacancy islands meet, but their internal dimer rows are not in the same registry. In contrast to $\mathrm{Si}(001)$, where only one type of antiphase boundary is found, we have found three different types of antiphase boundaries on $\mathrm{Ge}(001)$. Higher dose (> several monolayers) room temperature ion bombardment followed by annealing at temperatures in the range $400-500 \mathrm{~K}$ results in a surface which contains a high density of valleys. In addition to the preference for the annihilation of dimer vacancies at descending versus ascending steps we also suggest that the development of antiphase boundaries drives the roughening of this surface. Finally, several atomic rearrangement events, which might be induced by the tunneling process, are observed after low-dose ion bombardment at room temperature.
\end{abstract}

Keywords: Germanium; Ion bombardment; Low-index single-crystal surfaces; Scanning tunneling microscopy; Surface roughening

The evolution of surface morphology during non-equilibrium processes has been a topic of many experimental and theoretical papers. Crystal growth is probably the most widely studied nonequilibrium surface process, partly due to the importance of the interface structure to the synthesis of multi-layer thin film devices. The related problem presented by the etching of crystals, by for example ion bombardment, has attracted less attention [1,2] despite the critical role etching processes play in the fabrication of microelectronic structures and microanalysis. Using scanning tunneling microscopy (STM) the surface morphology can nowadays be studied on an atomic scale. Recent diffraction [3] and scanning tunneling microscopy experiments [4] have revealed that $\mathrm{Si}$ under low-energy ion bombardment is mediated by mobile surface vacancies. There are a number of distinct differences between epitaxial growth and removal by ion bombardment. First, during ion bombardment both adatoms as well as vacancies are created, whereas during epitaxial growth adatoms are in most cases deposited one by one. Second, the vacancy and adatom clusters which develop immediately after the ion impact might even have a size larger than the critical nucleus size. Third, the created adatom and vacancy clusters can, owing to the ion impact, have an energy with exceeds the thermal energy $k T$. This increase in energy of the clusters enhances the intra- and interlayer mass transport and hence results, in general, in smoother surfaces.

Another complication, indissolubly related to the dimerisation of the $\mathrm{Ge}(001)$ surface, emerges 


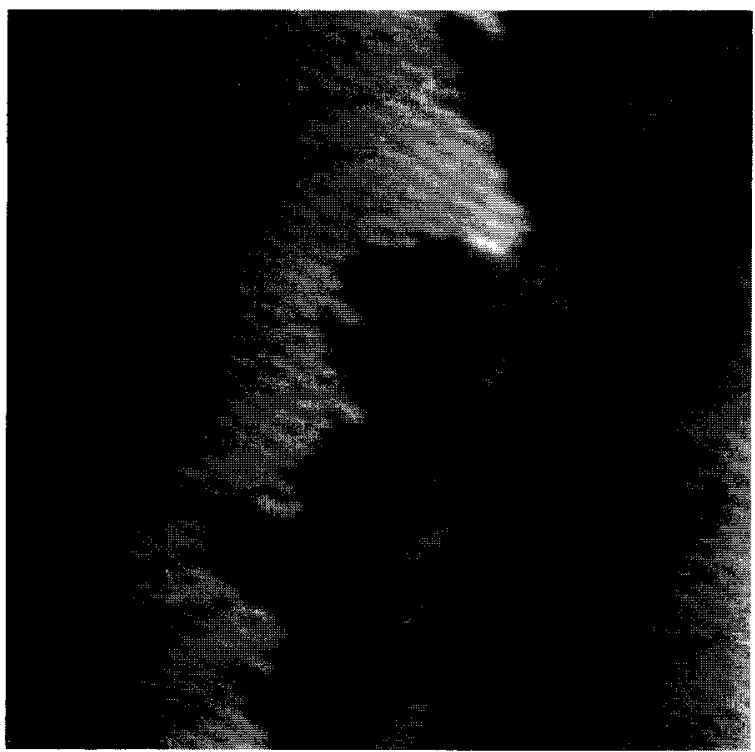

(a)

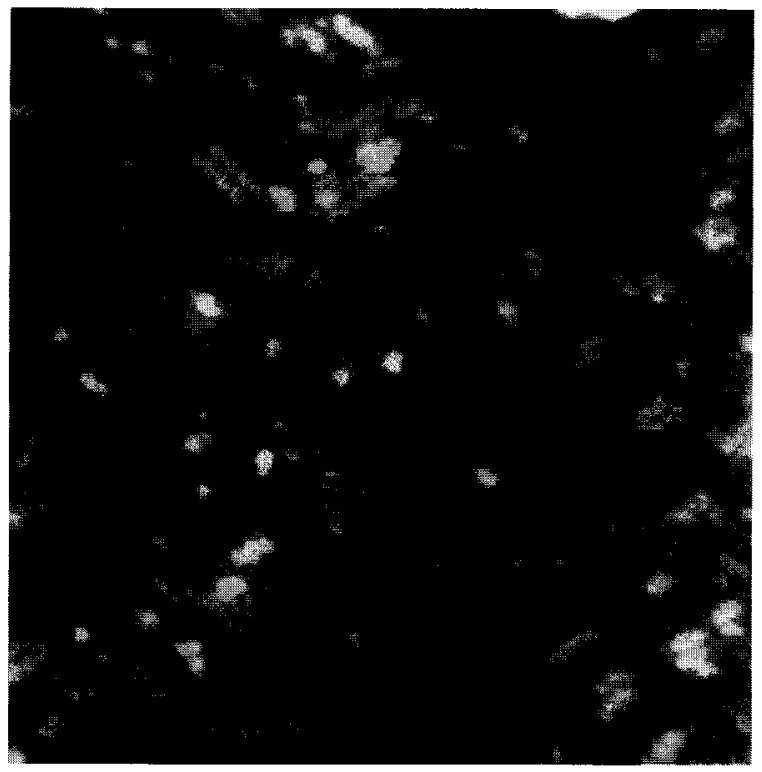

(c)

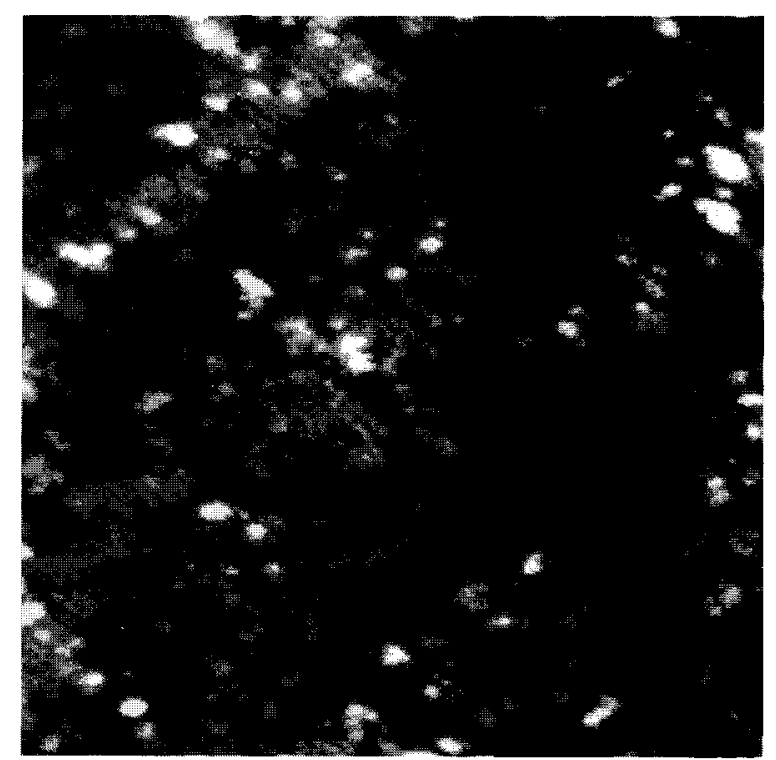

(b)

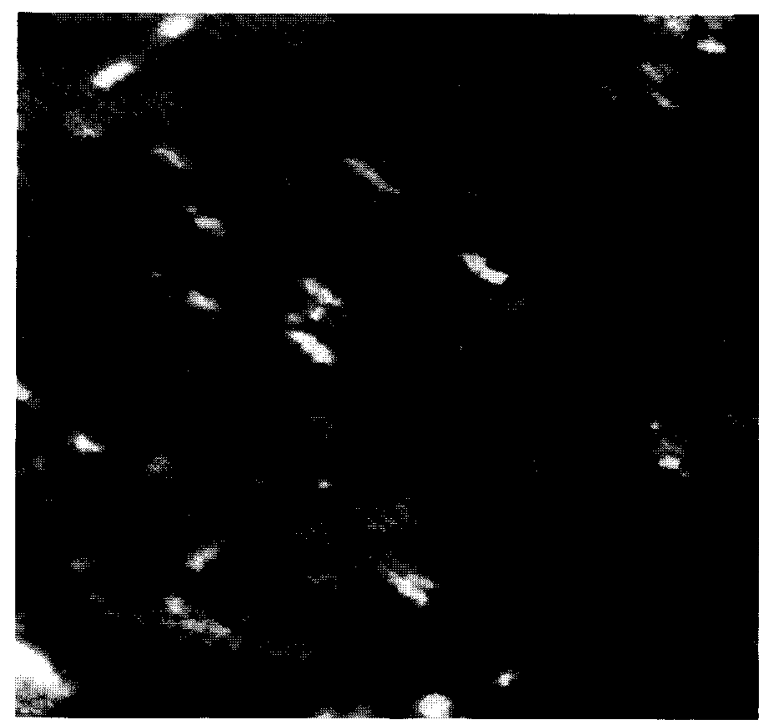

(d)

Fig. 1. STM images of (a) clean Ge(001) and (b-i) the same surface after $800 \mathrm{eV} \mathrm{Ar}+$ irradiation with a flux of $7 \times 10^{11}$ ions $\mathrm{cm}^{-2}$ $\mathrm{s}^{-1}$. The sample bias was $-2 \mathrm{~V}$ and the tunnel current was $1 \mathrm{nA}$. (a) Scan size $200 \times 300 \AA$. (b) Ge(001) surface after room temperature (RT) argon ion bombardment for $200 \mathrm{~s}$. Scan size $500 \times 500 \AA$ A. (c) As in (b) after a 2 min anneal at $380 \mathrm{~K}$. Scan size $500 \times 500 \AA$. (d) As in (c) after a 2 min anneal at $430 \mathrm{~K}$. Scan size $500 \times 500 \AA$. (e) As in (d) after a 2 min anneal at $500 \mathrm{~K}$. Scan size $500 \times 500 \AA$. (f) Ge(001) surface after RT argon ion bombardment for $2000 \mathrm{~s}$ and a 2 min anneal at $500 \mathrm{~K}$. Scan size $1000 \times 1000 \AA$. (g) $\mathrm{Ge}(001)$ surface after RT argon ion bombardment for $5000 \mathrm{~s}$ and 2 min anneal at $450 \mathrm{~K}$. Scan size $1000 \times 1000 \AA$. (h) Ge(001) surface after RT argon ion bombardment and 2 min anneal at $500 \mathrm{~K}$. Scan size $1000 \times 1000 \AA$ A. (i) Ge(001) surface after argon ion bombardment at a temperature of $400 \mathrm{~K}$ for $500 \mathrm{~s}$. Scan size $1000 \times 1000 \AA$. 


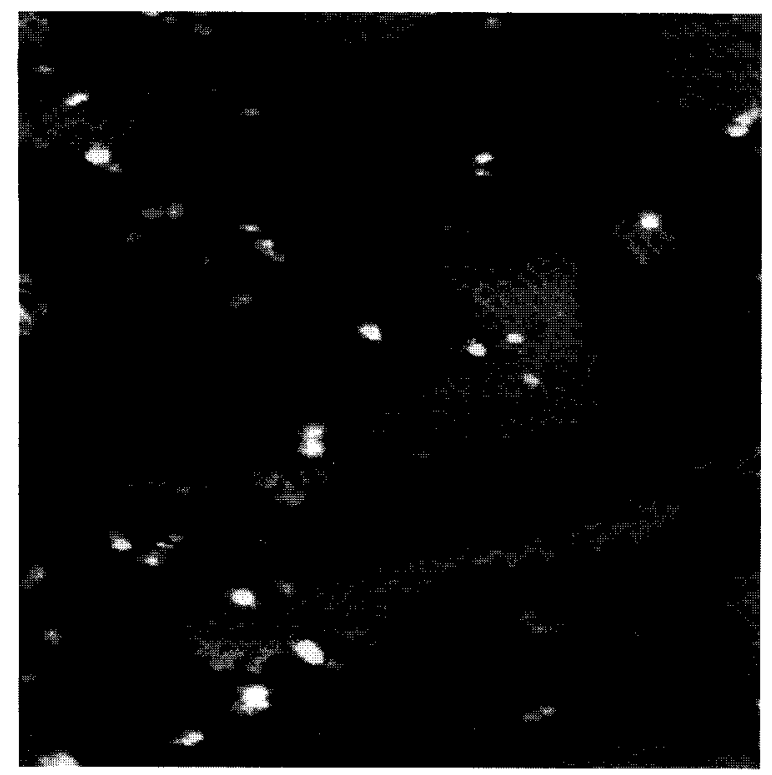

(e)

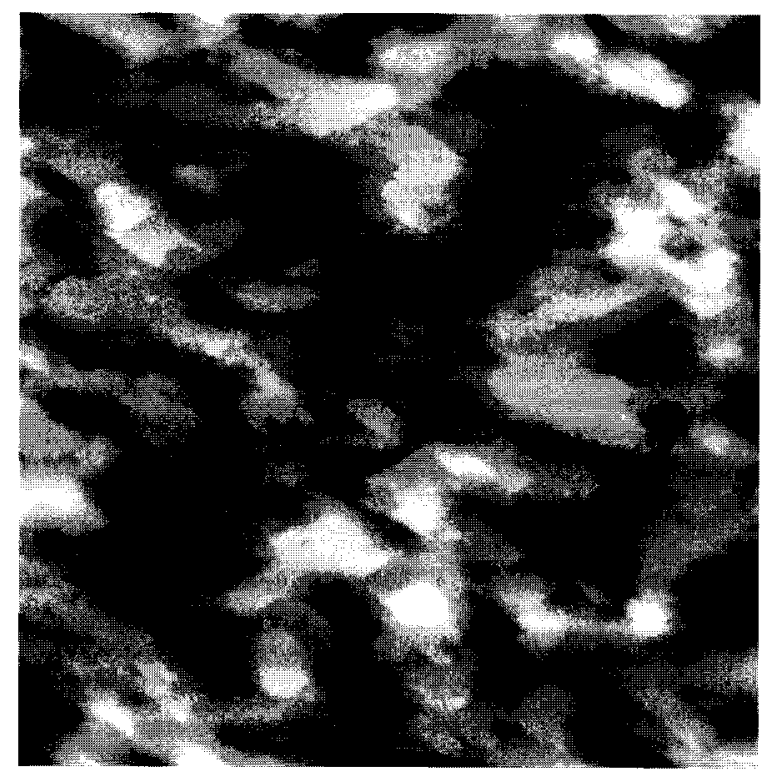

g)

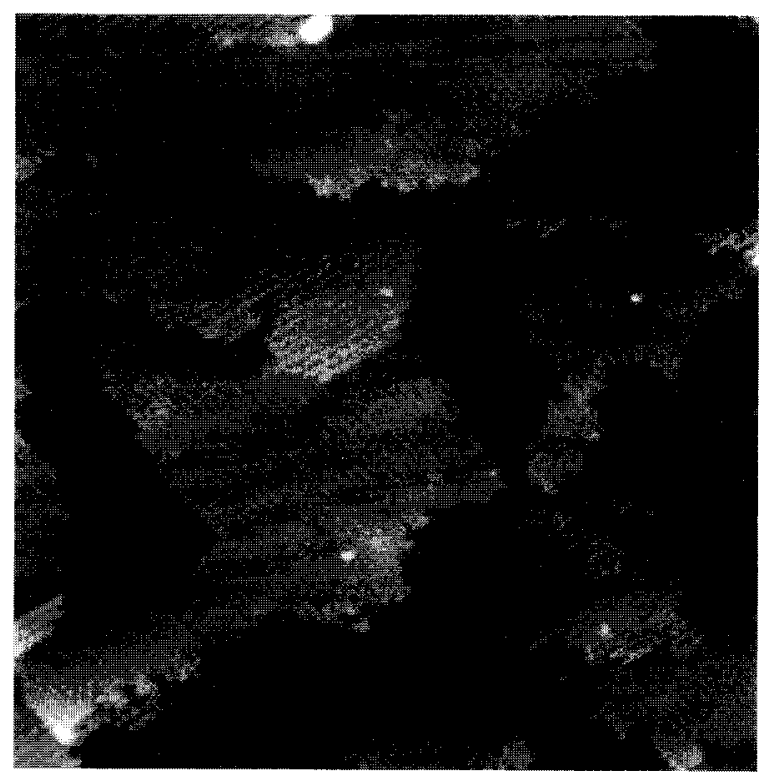

(f)

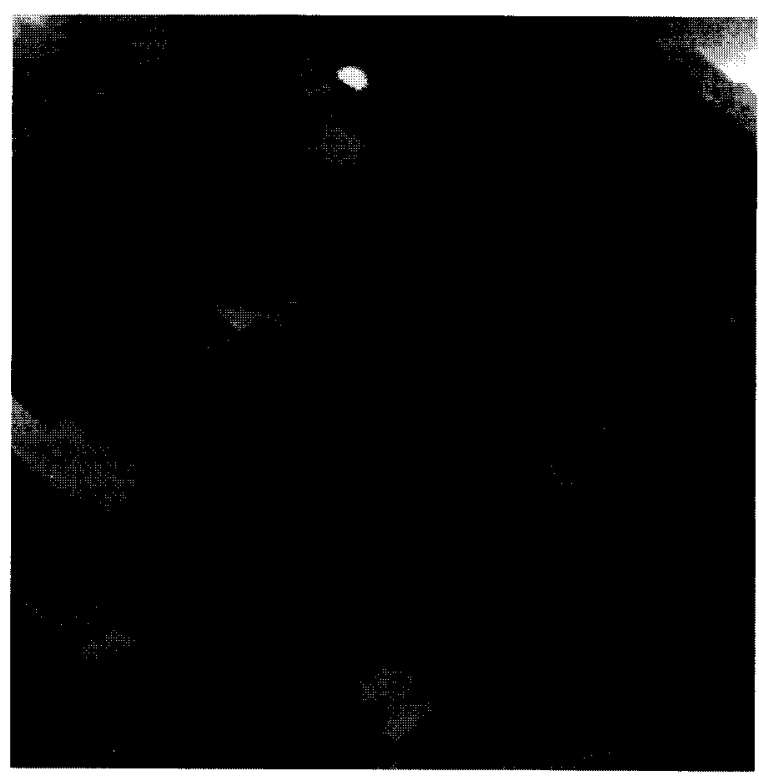

(h)

Fig. 1 (continued)

Juring the coalescence of the vacancy islands: as racancy islands nucleate and expand, the dimer ows in neighbouring vacancy islands do not have o be in the same registry because they nucleate ndependently. The coalescence of vacancy islands with an out-of-phase dimer row registry gives rise to an antiphase boundary, whereas an in-phase registry does not. Owing to the symmetry of the diamond lattice one expects, in principle, two different types of antiphase boundaries, i.e. along or perpendicular to the dimer row direction.

In this paper, we focus on the surface structure 


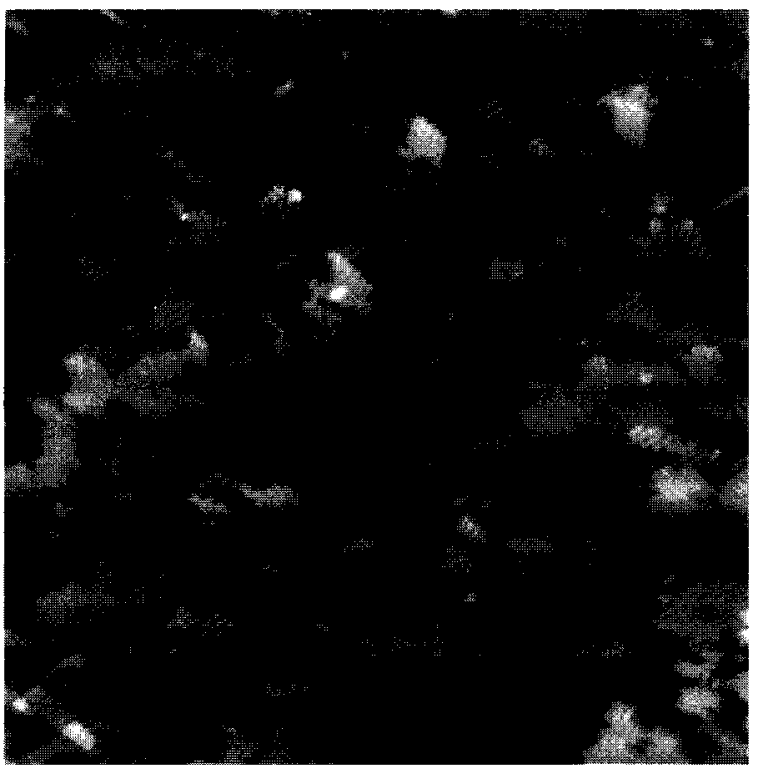

(i)

Fig. 1 (continued)

after low-energy ion bombardment. We have kept the flux and energy of the argon ions constant at $7 \times 10^{11}$ ions $\mathrm{cm}^{-2} \mathrm{~s}^{-1}$ and $800 \mathrm{eV}$, respectively; only the annealing temperature and the total dose are varied. Various types of antiphase boundaries are found after ion bombardment and subsequent annealing. We believe that the formation of these antiphase boundaries during ion bombardment drives, besides the preference for the annihilation of dimer vacancies at descending versus ascending steps [5], the roughening of the $\mathrm{Ge}(001)$ surface. Interestingly, Jay Chey et al. [5] found that the surface morphology during high-dose (up to 130 monolayers) etching with $240 \mathrm{eV}$ xenon ions changes from a relatively disordered arrangement of mounds $(T<545 \mathrm{~K})$ to a more regular pattern of valleys $(T>545 \mathrm{~K})$. Our low-dose ion bombardment experiments reveal that a valley-like pattern is already developed at temperatures as low as $400 \mathrm{~K}$. We never observed the mound-like pattern at low-dose removal (up to $5 \mathrm{ML}$ ) that was reported by Jay Chey et al. [5] for high-dose removal.

The ion bombardment and STM experiments were performed in an ultrahigh vacuum chamber with a base pressure $<1 \times 10^{-10}$ Torr. The system can be pumped with an ion pump integrated with a titanium sublimator and a turbo pump. The n-type $\mathrm{Ge}(001)$ samples with dimensions $0.3 \times 5 \times 10 \mathrm{~mm}^{3}$ were about $0.8^{\circ}$ misoriented towards [110]. After loading the samples in the UHV chamber they were outgassed at a temperature of about $700 \mathrm{~K}$ for several hours followed by several cycles of $\mathrm{Ar}^{+}$sputtering $\left(800 \mathrm{eV}, 2 \mu \mathrm{A} / \mathrm{cm}^{2}\right.$ for $30 \mathrm{~min}$ ) and annealing at $800-850 \mathrm{~K}$ for $15 \mathrm{~min}$. This procedure results in clean, nearly defect free $\mathrm{Ge}(001)$ surfaces. After cleaning and imaging with the STM the Ge(001) samples were bombarded with Argon ions at an energy of $800 \mathrm{eV}$ (normal incidence) and an ion current of about $100 \mathrm{nA} / \mathrm{cm}^{2}$. The number of removed monolayers varied from about 0.1 to $5 \mathrm{ML}$. After ion bombardment and subsequent annealing, the samples were transferred to the STM for imaging.

In Fig. 1a an STM image of a $\mathrm{Ge}(001)$ surface prior to ion bombardment is shown. In marked contrast to the clean $\mathrm{Si}(001)$ surface the $\mathrm{Ge}(001)$ surface is nearly defect free. After room temperature $800 \mathrm{eV}$ argon ion bombardment at a flux of $7 \times 10^{11}$ ions $\mathrm{cm}^{-2} \mathrm{~s}^{-1}$ for $200 \mathrm{~s}$, resulting in the removal of about $0.2 \mathrm{ML}$, both vacancy and adatom islands are resolved in Fig. 1b. The bombarded surface in Fig. 1b looks a little disordered but subsequent annealing results in a decrease of the disorder. The vacancy islands are only one monolayer deep and high resolution images reveal that the atoms within the vacancy islands are dimerized. The elongation of the vacancy island in the direction parallel to the dimer rows of the upper terrace is consistent with previous observations on $\mathrm{Si}(001)$ and has been shown to result from preferential annihilation of mobile surface vacancies at the ends, rather than the sides, of the dimer rows [4]. There is, however, one remarkable difference with the $\mathrm{Si}(001)$ surface: low-dose (vacancy concentration $<0.2 \mathrm{ML}$ ) sputtering of $\mathrm{Si}(001)$ by $3 \mathrm{keV}$ argon ions and subsequent annealing at sufficiently high temperatures $(>800 \mathrm{~K})$ was found to introduce line defects which are oriented perpendicular to the dimer rows of the upper terrace $[6,7]$. We have never found such line defects on $\mathrm{Ge}(001)$ after low-dose sputtering and annealing. Small differences in the anisotropic vacancy island edge formation energy and surface stress tensor might explain this different behaviour. 
The number density of adatom and vacancy islands decreases, whereas the averaged vacancy island size and spacing increases with increasing anneal temperature (see Figs. 1c-1e). These observations imply that intralayer transport of vacancies and adatom clusters must occur at temperatures as low as $380 \mathrm{~K}$. Annealing at temperatures above $600 \mathrm{~K}$ is sufficient to restore the original surface morphology. In Figs. 1f-1h the total removed dose is increased from $2 \mathrm{ML}$ (Fig. 1f) to $5 \mathrm{ML}$ (Figs. 1g and $1 \mathrm{~h}$ ). The characteristic network of valleys with depths up to $10 \mathrm{ML}$ can clearly be resolved in Fig. 1g. Annealing of this rough surface for $2 \mathrm{~min}$ at $500 \mathrm{~K}$ already results in a significant amount of smoothening. Jay Chey et al. [5] proposed that the valley-like pattern might be due to a preference for vacancy annihilation at descending steps versus ascending steps. We agree with these authors on this point but we believe that also the formation of antiphase boundaries (discussed in detail below) may play a role in this roughening process. In Fig. 1i a $\mathrm{Ge}(001)$ surface bombarded at $400 \mathrm{~K}$ by $800 \mathrm{eV}$ argon ions is shown, the total removed dose is about $0.5 \mathrm{ML}$ and the flux is again $7 \times 10^{11}$ ions $\mathrm{cm}^{-2} \mathrm{~s}^{-1}$. The number density of adatom clusters after the $400 \mathrm{~K}$ bombardment experiment is much lower as compared to the surface bombarded at room temperature followed by annealing at $400 \mathrm{~K}$. This low number density of adatom clusters suggests that interlayer transport and adatom-vacancy annihilation does occur at sputtering temperatures above $400 \mathrm{~K}$. Probably, the adatom and vacancy clusters created at elevated temperatures have an additional energy component due to the ion impact which enhances the mass transport process at the surface. In any event, we must conclude that ion bombardment at elevated temperatures is not equivalent to ion bombardment at room temperature followed by annealing at the elevated temperature. Despite the fact that much higher ion bombardment energy (several keVs) and fluxes were used in the work of Hoeven et al. [2] several observations in their work, in particular the persistence of the reflection high energy electron diffraction (RHEED) pattern during ion sputtering at elevated temperatures, are in agreement with our results.

Further annealing of the bombarded surface

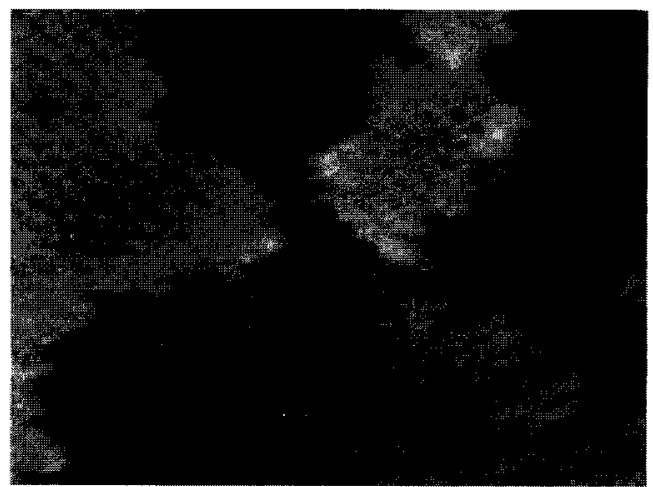

(a)

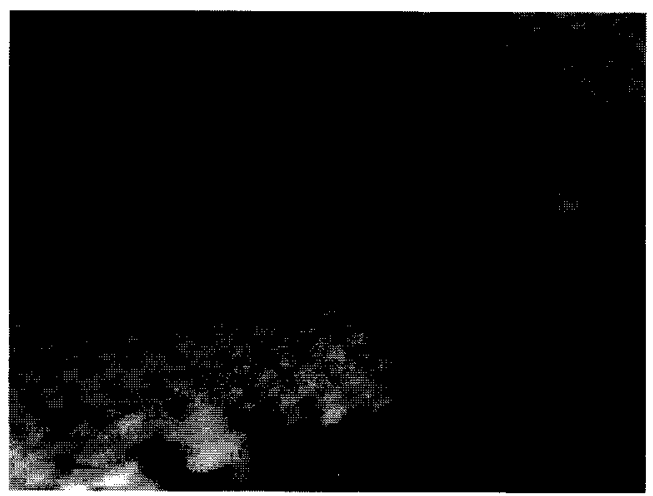

(b)

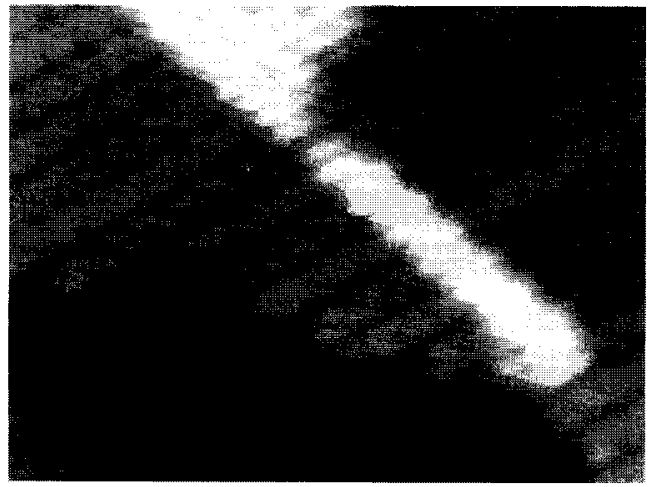

(c)

Fig. 2. Three different types of antiphase boundaries on the $\mathrm{Ge}(001)$ surface after $800 \mathrm{eV}$ argon ion bombardment and subsequent annealing. Sample bias $-2 \mathrm{~V}$ and tunnel current $1 \mathrm{nA}$.

leads finally to the coalescence of neighbouring vacancy islands. The vacancy islands nucleate separately and independently and the dimerization within the vacancy islands has a probability of $50 \%$ to be in the right (or wrong) registry. If the 
dimer rows in the two neighbouring vacancy islands do not align then an antiphase boundary will develop. In Fig. 2 three different types of antiphase boundaries are displayed. In Fig. 2a there is an undecorated antiphase boundary in the dimer row direction, whereas in Fig. $2 b$ an undecorated antiphase boundary positioned in a direction perpendicular to the dimer rows is present. The buckling registry of the dimers in the two dimer rows which form the antiphase boundary in Fig. 2b are in phase along the antiphase boundary direction. In Fig. $2 \mathrm{c}$ a decorated antiphase boundary, which is similar to the antiphase boundary on $\mathrm{Si}(001)$, is displayed. On $\mathrm{Si}(001)$ only one type antiphase boundary has been reported by Bedrossian and Kaxiras [8]. In the case of $\mathrm{Si}(001)$ these decorated antiphase boundaries are as narrow as one dimer row and as long as the substrate's terrace width. On $\mathrm{Ge}(001)$, two of the three types of antiphase boundaries are not decorated with a dimer row. Annealing above $600 \mathrm{~K}$ removes these antiphase boundaries completely. Especially, the "zipper" type of antiphase boundary (Fig. 2c) is very stable. The high stability of this type of antiphase boundary as well as the preference of vacancies to annihilate at descending steps
[5] might explain the development of the valleylike surface morphology as shown in Fig. 1g. Also, during $\mathrm{Si}$ homoepitaxial growth the $\mathrm{Si}$ islands nucleate independently and, when growing islands meet, the dimer row registry can be in phase or out of phase $[9,10]$. Hamers et al. $[9,10]$ showed that these antiphase boundaries act as preferential nucleation sites, in perfect agreement with Bedrossian and Kaxiras' experimental and theoretical findings [8] on $\mathrm{Si}(001)$ as well as with our experimental data of $\mathrm{Ge}(001)$. Interestingly, the antiphase boundary depicted in Fig. 2 a cannot compared with the AP2-type antiphase boundary (notation of Hamers et al. [9]) found during homoepitaxial growth by Hamers et al. [9], whereas the antiphase boundary in Fig. $2 b$ is the same as the AP1-type antiphase boundary [9]

Finally, it is interesting to note that low-dose ion bombardment at room temperature induced some damage which seems to anneal away at room temperature (although it cannot be ruled out that these rearrangement events are induced by the tunneling process). The sample bias was kept at $-2 \mathrm{~V}$ and the tunnel current was kept fixed at $1 \mathrm{nA}$ for all images. In Fig. 3 four subsequent images of a small part of the $\mathrm{Ge}(001)$ surface are

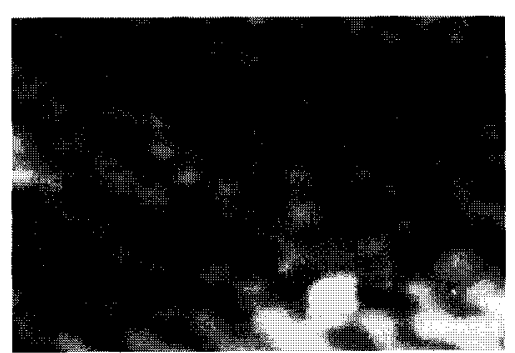

(a)
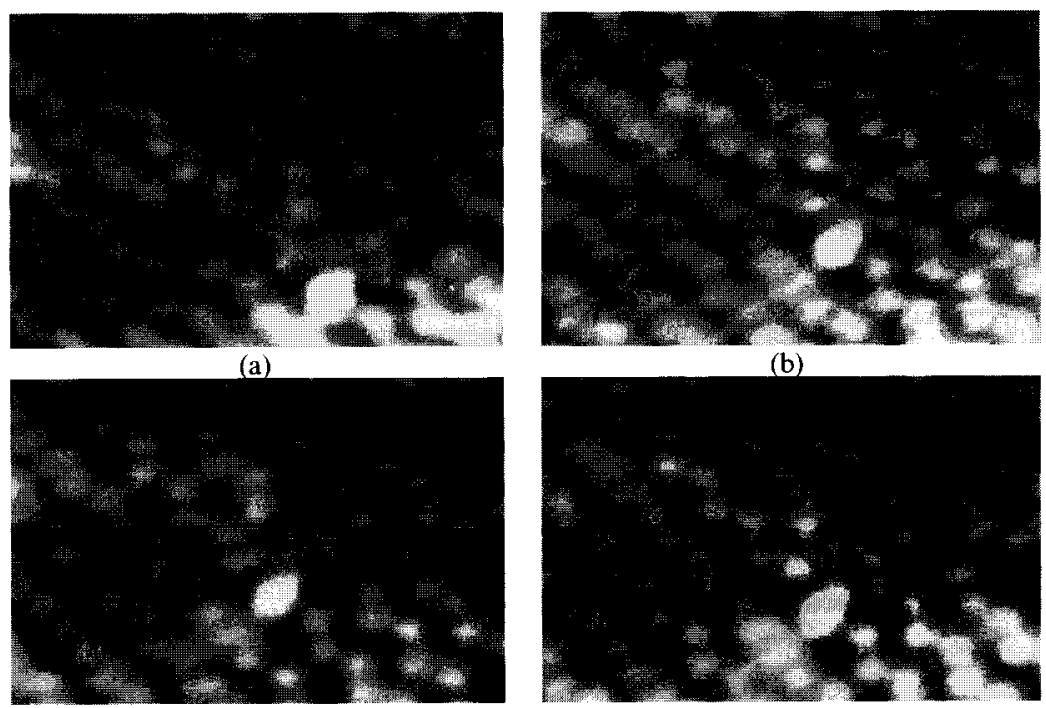

(c)

(d)

Fig. 3. Four subsequent STM images of a Ge(001) surface after low dose $800 \mathrm{eV}$ argon bombardment at room temperature. The buckling of the dimer row where the ad-dimer is positioned as well as the adjacent dimer row changes from image to image. The local registry changes between $c(4 \times 2)$ and $\mathrm{p}(2 \times 2)$ and vice versa. Sample bias $-2 \mathrm{~V}$ and tunnel current $1 \mathrm{nA}$. 


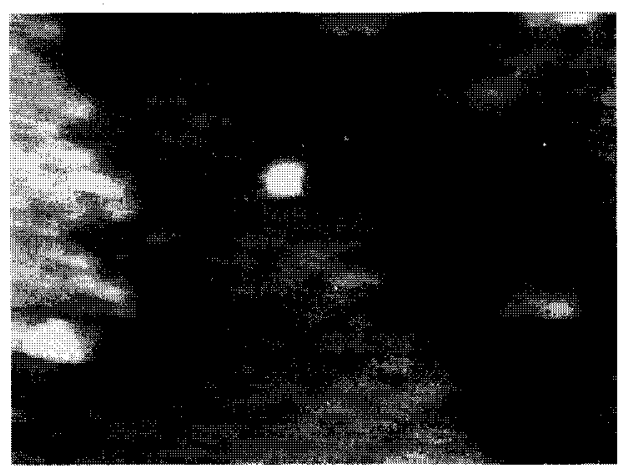

(a)

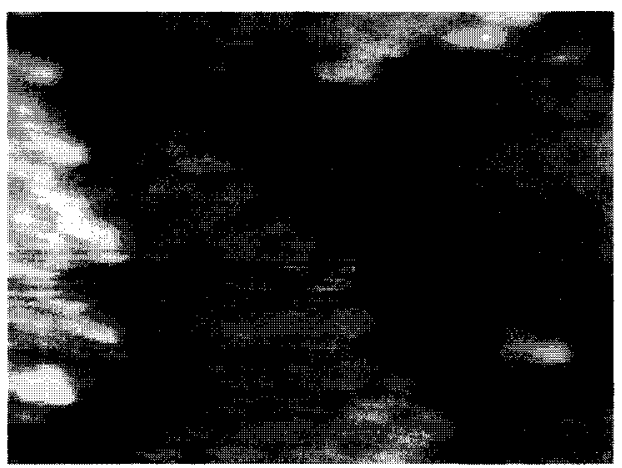

(b)

Fig. 4. Two subsequent STM images of a Ge(001) surface after low dose $800 \mathrm{eV}$ argon bombardment at room temperature. The white blob (an ad-dimer) disappears in the second image. Sample bias $-2 \mathrm{~V}$ and tunnel current $1 \mathrm{nA}$.

shown. In the dimer row where the ad-dimer is positioned and the adjacent dimer row the local buckling registry changes locally from $\mathrm{c}(4 \times 2)$ in Fig. $3 \mathrm{a}$, to $\mathrm{p}(2 \times 2)$ in Fig. $3 \mathrm{~b}$ to $\mathrm{c}(4 \times 2)$ in Fig. $3 \mathrm{c}$ and finally to $\mathrm{p}(2 \times 2)$ in Fig. 3d. In Fig. $4 a$ an ad-dimer is present near a large defect, whereas it has disappeared in the next image. It is remarkable to note is that we were not able to observe any such rearrangement events on the unbombarded Ge(001) surface using the same tunneling conditions. This does not necessarily mean that the rearrangement events on the bombarded sample are not induced by the STM tip, because the local arrangement of bombarded areas and the corresponding diffusion and rearrangement barriers may be different.

In conclusion, we have studied the surface morphology of the $\mathrm{Ge}(001)$ surface after low-energy ion bombardment. We have found that the vacancy islands are always elongated in a direction along the dimer row direction of the upper terrace. Furthermore, we have observed three different types of antiphase boundaries. The surface damage and antiphase boundaries can be annealed away after annealing at temperatures above $600 \mathrm{~K}$. We believe that besides the preference for the annihilation of defects at descending steps versus ascending steps the development of antiphase boundaries drives the roughening of this surface. Finally, it seems that some of the ion bombardment induced damage can be annealed away at room temperature, although we were not able to rule out the influence of the tunneling process on these rearrangement events or the changes in the buckling registry.

\section{References}

[1] D.E. Aspnes and A.A. Studna, Surf. Sci. 96 (1980) 294.

[2] A.J. Hoeven, J.S.C. Kools, J. Aarts and P.C. Zalm, in: Reflection High Energy Electron Diffraction and Reflection Electron Imaging of Surfaces, Eds. P.K. Larsen and P.J. Dobson (Plenum, New York, 1988)

[3] P. Bedrossian, J.E. Houston, J.Y. Tsao, E. Chason and S.T. Picraux, Phys. Rev. Lett. 67 (1991) 124.

[4] P. Bedrossian and T. Klitsner, Phys. Rev. Lett. 68 (1992) 646.

[5] S. Jay Chey, J.E. Van Nostrand and D.G. Cahill, Phys. Rev. B 52 (1995) 16696.

[6] H.J.W. Zandvliet, H.B. Elswijk, E.J. van Loenen and I.S.T. Tsong, Phys. Rev. B 46 (1992) 7581.

[7] H. Feil, H.J.W. Zandvliet, M.-H. Tsai, J.J. Dow and I.S.T. Tsong, Phys. Rev. Lett. 69 (1992) 3076.

[8] P. Bedrossian and E. Kaxiras, Phys. Rev. Lett. 70 (1993) 2589

[9] R. Hamers, U. Köhler and J. Demuth, J. Vac. Sci. Technol. A 8 (1990) 195.

[10] R. Hamers, U. Köhler and J. Demuth, Ultramicroscopy 31 (1989) 10. 